\title{
Reibungsverhalten nasslaufender Lamellenkupplungen am Übergang Haftreibung - Gleitreibung
}

\author{
Katharina Völkel ${ }^{1} \mathbb{D} \cdot$ Georg Johann Meingaßner ${ }^{1} \mathbb{D} \cdot$ Hermann Pflaum $^{1} \mathbb{D} \cdot$ Karsten Stahl $^{1} \mathbb{D}$
}

Eingegangen: 19. Mai 2020 / Angenommen: 4. Januar 2021 / Online publiziert: 18. Januar 2021

(c) Der/die Autor(en) 2021

\section{Zusammenfassung}

Nasslaufende Lamellenkupplungen übernehmen in industriellen und automobilen Antriebssträngen meist sicherheits- und komfortrelevante Funktionen. Kenntnisse zum übertragbaren Drehmoment bei den entsprechenden Belastungen und Betriebsbedingungen sind die Basis für eine betriebssichere Auslegung und Dimensionierung der Lamellenkupplung. In einigen Anwendungsfällen wird trotz konservativer Dimensionierung ein unerwünschtes Kriechen der Lamellenkupplung bei langer und häufig stationärer Drehmomentbelastung festgestellt, wodurch Funktion und Sicherheit beeinträchtigt sein können.

Zum Reibungsverhalten bei Mikroschlupf und der ohne Schlupf übertragbaren Reibungszahl liegen bisher nur wenige Erkenntnisse vor. Im Rahmen grundlegender experimenteller Untersuchungen wurden deswegen das Reibungsverhalten bei Mikroschlupf sowie die übertragbare Haftreibungszahl untersucht. Es wurde eine Test- und Auswertemethodik entwickelt und in experimentellen Untersuchungen exemplarisch an einer Kupplung aus einer Automatikgetriebe-Anwendung umgesetzt. Die Methodik ermöglicht eine Untersuchung des Reibungsverhaltens nasslaufender Lamellenkupplungen bei sehr niedrigen Gleitgeschwindigkeiten und die Einordnung zum Lastschalt-Reibungsverhalten.

\section{Friction behavior of wet multi-plate disk clutches at the transition from static to dynamic friction}

\begin{abstract}
Wet multi-plate clutches are used in industrial and automotive powertrains mainly for safety and comfort tasks. Knowledge on the transmissible torque depending on load and operating conditions is essential for reliable dimensioning of the clutch. In some applications creeping and microslip of the clutch can be noticed under long-term and stationary torque load which can affect function and safety.

Currently, there is only a little understanding of the friction behavior of disk clutches under microslip conditions as well as the transmittable static torque without slip. Therefore, basic experimental investigations on the friction behavior under microslip conditions and the static coefficient of friction were carried out. A test and evaluation method has been developed and implemented in experimental studies on a clutch from an automatic transmission application. The method enables evaluation of friction behavior of wet disk clutches under low sliding speeds as well as a classification with regard to the powershift friction behavior.
\end{abstract}

The data that support the findings of this study are available from the Research Association for Drive Technology e. V. (FVA) but restrictions apply to the availability of these data, which were used under license for the current study, and so are not publicly available. Data are however available from the authors upon reasonable request and with permission of FVA.
Katharina Völkel

voelkel@fzg.mw.tum.de

$\triangle$ Hermann Pflaum

pflaum@fzg.mw.tum.de

1 Forschungsstelle für Zahnräder und Getriebebau (FZG), Technische Universität München, Boltzmannstr. 15, 85748 Garching, Deutschland 


\section{Einleitung und Problemstellung}

Nasslaufende Lamellenkupplungen finden sehr weite Verbreitung in Fahrzeuggetrieben und Industrieanwendungen und übernehmen meist sicherheits- und komfortrelevante Funktionen. In Automatikgetrieben stellen sie eine der wichtigsten Baugruppen dar und werden zunehmend auch schlupfgeregelt betrieben.

Die hohen Anforderungen an Komfort und Regelbarkeit und die dafür vorteilhafte Einstellung einer mit der Gleitgeschwindigkeit ansteigenden Reibungszahl führen dazu, dass die Kupplungen im geschlossenen Zustand häufiger an der Grenze ihrer Drehmoment-Übertragungsfähigkeit betrieben werden. So wird in einigen Anwendungsfällen trotz konservativer Dimensionierung ein unerwünschtes Kriechen (Mikroschlupf) der Lamellenkupplung bei langer und stationärer Drehmomentbelastung festgestellt [1]. Dadurch können Funktion und Sicherheit erheblich beeinträchtigt werden. Auch Schäden wie Verschleiß und Veränderungen im Reibungsverhalten werden mitunter in Verbindung mit unerwünschten Drehzahlschlupf gebracht [2]. In der Praxis muss bei derartigen Problemen mit verschleißfesteren und thermisch stabileren Kupplungsbelägen nachgebessert werden. Mögliche Folgen sind dabei eine Verschlechterung der dynamischen Reibeigenschaften sowie eine Überdimensionierung der Kupplungen.

Unerwünschter Schlupf kann aber auch als Folge von äußeren Zwangskräften beispielsweise aus Winkel- oder Achsversatz von An- und Abtriebswelle oder aufgrund von Schwingungen durch Drehungleichförmigkeiten auftreten (u. a. [3-5]).

Zum Reibungsverhalten bei Mikroschlupf und der ohne Schlupf übertragbaren Reibungszahl liegen aktuell nur wenige Erkenntnisse vor. Im Rahmen grundlegender experimenteller Untersuchungen wurden deshalb das Reibungsverhalten bei Mikroschlupf sowie die übertragbare Haftreibungszahl untersucht. Dafür wurde eine geeignete Testund Auswertemethodik entwickelt und in experimentellen Untersuchungen exemplarisch an einer Kupplung aus einer Automatikgetriebe-Anwendung umgesetzt.

\section{Reibungsverhalten bei niedrigen Gleitgeschwindigkeiten}

Nasslaufende Lamellenkupplungen werden hauptsächlich im Bereich der Grenzreibung betrieben. Damit ist das Reibungsverhalten sehr stark vom Reibsystem und damit von Grundöl und Additivierung (vgl. z.B. [6-8]), Reibbelag (vgl. z.B. [9-12]) und Stahllamelle (vgl. z. B. [13-16]) abhängig. In der Literatur werden basierend auf empirischen Beobachtungen neben der Reibmaterial-Schmierstoffpaa- rung zudem vielfältige weitere Einflüsse auf das Reibungsverhalten beschrieben:

- Gleitgeschwindigkeit und Gleitbeschleunigung (vgl. z.B. $[17,18])$

- zurückgelegter Gleitweg (vgl. z. B. [19, 20])

- Temperatur (vgl. z. B. [21, 22])

- Flächenpressung der in Kontakt stehenden Körper (vgl. z.B. $[23,24])$

Aufgrund der komplexen Wechselwirkungen im tribologischen System können Daten zur übertragbaren Reibungszahl bisher nur experimentell bestimmt werden. Hierfür werden häufig Untersuchungen an Komponentenprüfständen durchgeführt (u. a. [13, 25, 26]).

Im Hinblick auf Schlupfbeanspruchungen führte [27] Untersuchungen an einer Allrad-Differenzial-Kupplung mit sintermetallischem Reibbelag durch. Der Versuchsablauf zeigte einen großen Einfluss auf das Reibungsverhalten, was auf unterschiedliche Oberflächentemperaturen beim Start des Versuchs zurückgeführt wird. Als Haupteinflussparameter auf die Reibungszahl bei Schlupfbeanspruchung wurden Gleitgeschwindigkeit, Kontakttemperatur, Flächenpressung und Additivierung identifiziert.

[7] und [18] erklären eine mit sinkender Relativgeschwindigkeit sinkende Reibungszahl durch die Scherfestigkeit der Grenzschichten, die mit organischen Friction Modifier Additiven in ATFs erzeugt werden. Das Reibungsverhalten kann zudem durch Detergent- und DispersantAdditive durch eine lokale Erhöhung der Viskosität des Flüssigkeitsfilms nahe der Reibflächen beeinflusst werden, wodurch die Reibungszahl ansteigt.

Anhand von Tribometerversuchen („Pin-on-Disk“) werden in [13] stationäre Schlupfversuche mit kurzer Schlupfdauer und dynamischer Beschleunigung und Verzögerungsphase durchgeführt. Neben einer signifikanten Abhängigkeit der Reibungszahl von der Gleitgeschwindigkeit wird eine Abhängigkeit des Reibungszahlniveaus von der Flächenpressung festgestellt. Vergleichbare Ergebnisse für den Einfluss der Flächenpressung papierbasierter Reibwerkstoffe werden auch in [28] mittels Tribometerversuchen erzielt. Dabei werden die Auswirkungen einer thermischen Überlastung des Reibmaterials untersucht. Ohne Additivierung zeigen die Messungen einen über der Gleitgeschwindigkeit stark negativen Reibungszahlgradienten. Die untersuchten Gleitgeschwindigkeiten werden nicht genannt.

Im Hinblick auf das Reibungsverhalten bei niedrigen Gleitgeschwindigkeiten wird in [29] und [30] eine standardisierte Prüfprozedur zur Ermittlung des Reibungsverhaltens auf Basis von Schaltversuchen beschrieben. Die statische Reibungszahl wird dabei über Schaltversuche bei einer ,Abfang-/Enddrehzahl“ von $\mathrm{n}=2,0 \mathrm{~min}^{-1}$ (bzw. Weiterschleppen nach Schaltvorgang mit $\omega=0,21 \mathrm{rad} / \mathrm{s}$ ) ermittelt. 
[31] beschreibt einen Prüfstand zur Ermittlung der Haftreibungszahl sowie zur Untersuchung von Lamellenkupplungen bei Langzeitbelastung. Eine gegenüber der dynamischen Reibungszahl zu niedrige Haftreibungszahl kann in der Praxis zu kontinuierlichem Schlupf mit hoher thermischer Beanspruchung führen. Der Brems-Prüfstand besteht aus einem an der Antriebswelle befestigten Hebel, über den mittels Gewichten ein Drehmoment aufgebracht werden kann. Die Abtriebswelle ist über eine Drehmomentmesswelle mit dem Prüfstandsgehäuse befestigt. Es werden keine Versuchsergebnisse gezeigt.

\section{Prüfstandstechnik}

Im Rahmen der Untersuchungen kommen zwei Komponenten-Prüfstände der FZG zum Einsatz. Für ausführliche Beschreibungen der beiden Prüfstände wird auf [25, 32, 33], verwiesen.

\subsection{Prüfstand ZF/FZG KLP-260}

Für die Untersuchungen bei definiertem Kupplungsschlupf kommt der Prüfstand ZF/FZG KLP-260 zum Einsatz (Abb. 1).

Über den zuschaltbaren Kriechantrieb können variabel Konstant-Schlupf oder instationäre Schlupfverläufe mit und ohne Drehrichtungswechsel dargestellt werden. Der Inkrementalgeber am Antrieb ermöglicht eine genaue Erfassung des Schlupfs. Über die Messtechnik können u. a. Reibmoment und Reibungszahl unter definierten Betriebsbedingungen gemessen werden.

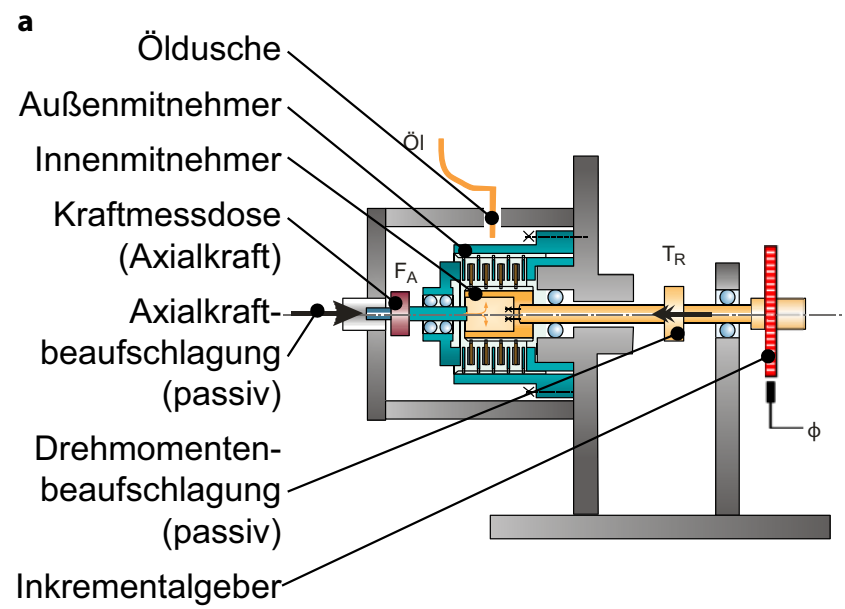

b

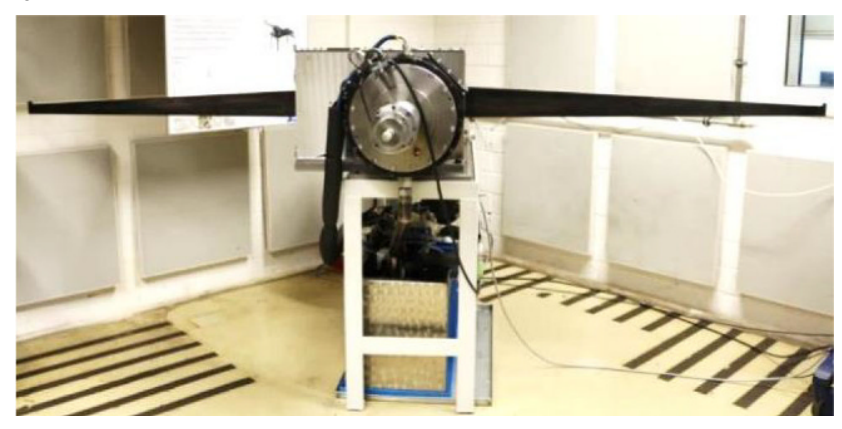

Abb. 2 Prüfstand LK3 (statisch) [32, 33]. a Schema, b Foto

\subsection{Prüfstand LK3 (statisch)}

Die Untersuchungen bei definierter Drehmomentbelastung werden am Prüfstand LK3 (statisch) durchgeführt (Abb. 2).

Der passive Aufbau ermöglicht die Aufbringung einer über lange Zeit konstanten Anpresskraft, die über eine Kraftmessdose gemessen wird. Das Lastmoment wird über a

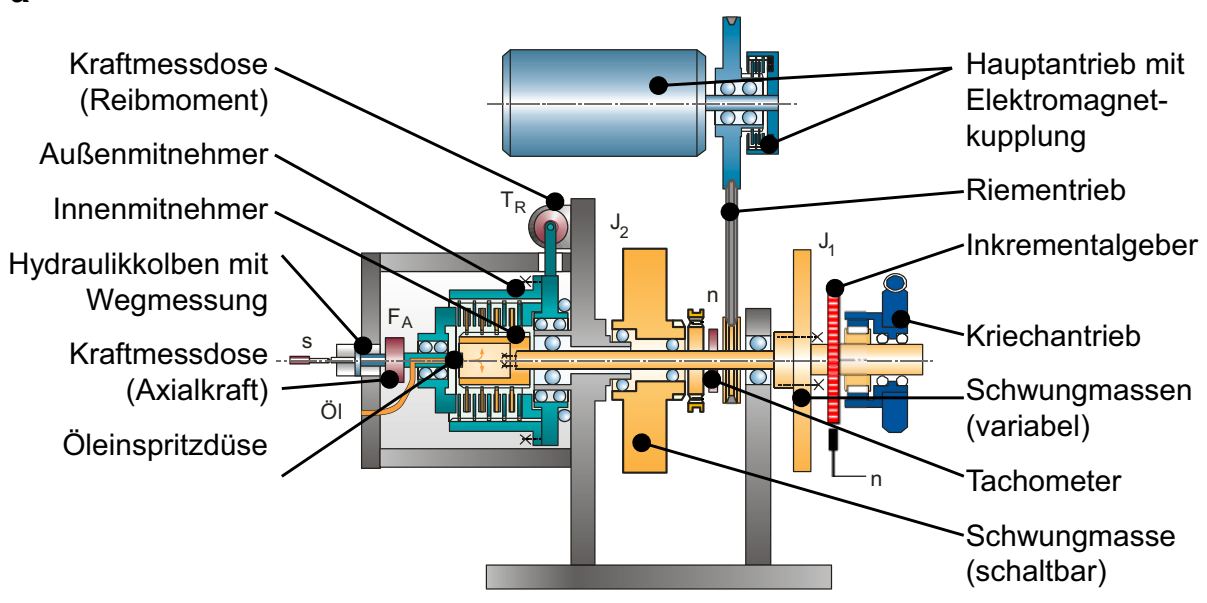

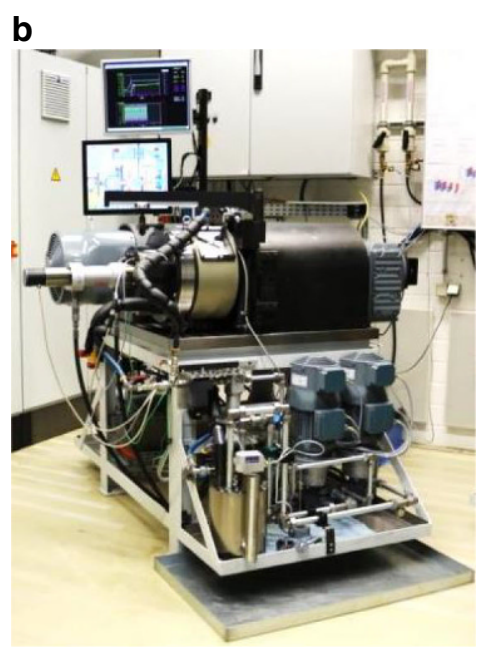

Abb. 1 Prüfstand ZF/FZG KLP-260 [25, 32, 33]. a Schema, b Foto 


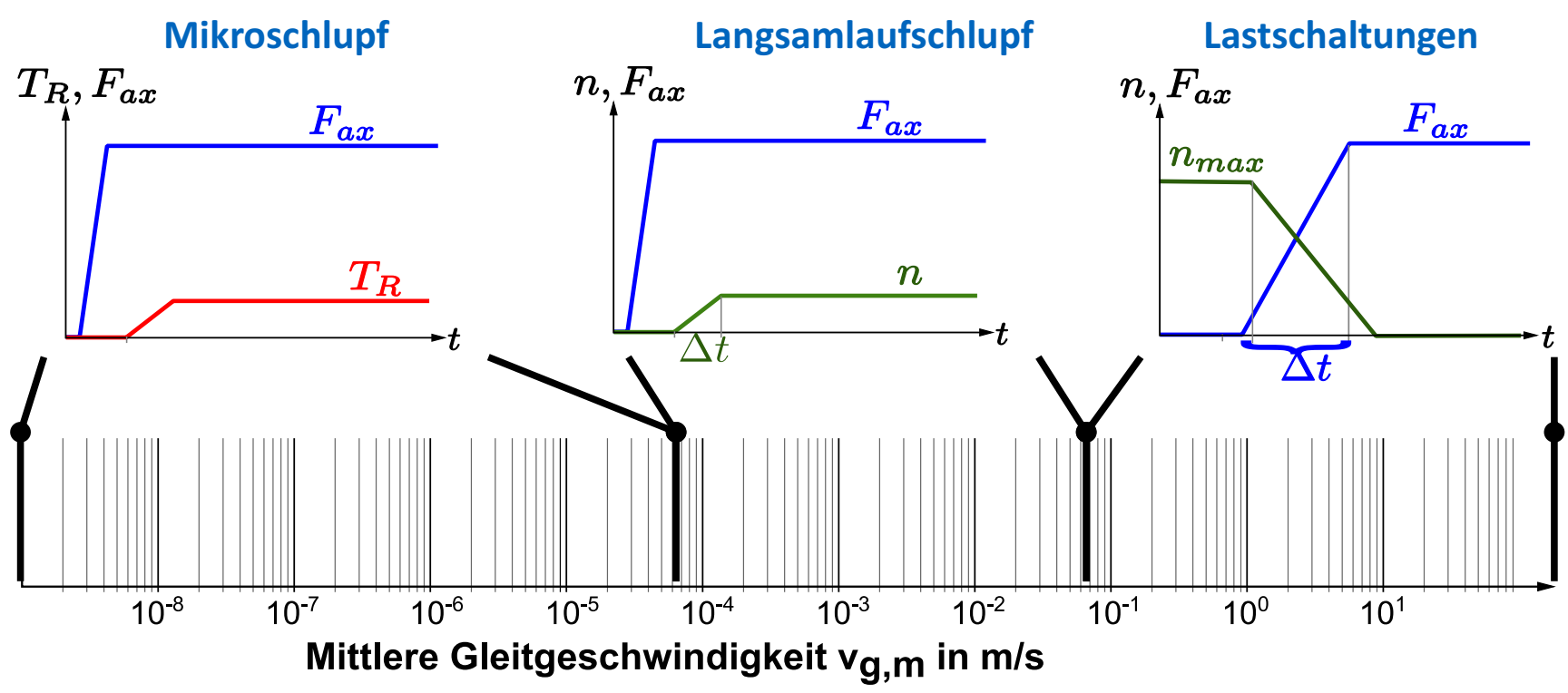

Abb. 3 Betriebsarten und Gleitgeschwindigkeitsbereiche - Übersicht [32]

einen Hebel und Gewichte in das Lamellenpaket eingeleitet. Der auftretende Schlupf (Verdrehwinkel) kann über den Inkrementalgeber sehr genau erfasst werden. Über ein Ölaggregat sind Beölung und Temperierung des Versuchspakets sichergestellt.

\section{Versuchs- und Auswertemethodik}

Eine wichtige Beurteilungsgröße für das Reibungsverhalten einer Lamellenkupplung stellt die Reibcharakteristik dar (u. a. [6, 31-34]). Als Reibcharakteristik wird der Verlauf der Reibungszahl über der Gleitgeschwindigkeit bezeichnet. Hinsichtlich Regelbarkeit und Schaltkomfort sowie zur Reduzierung der Reibschwingneigung wird eine mit zunehmender Gleitgeschwindigkeit ansteigende Reibungszahl angestrebt.

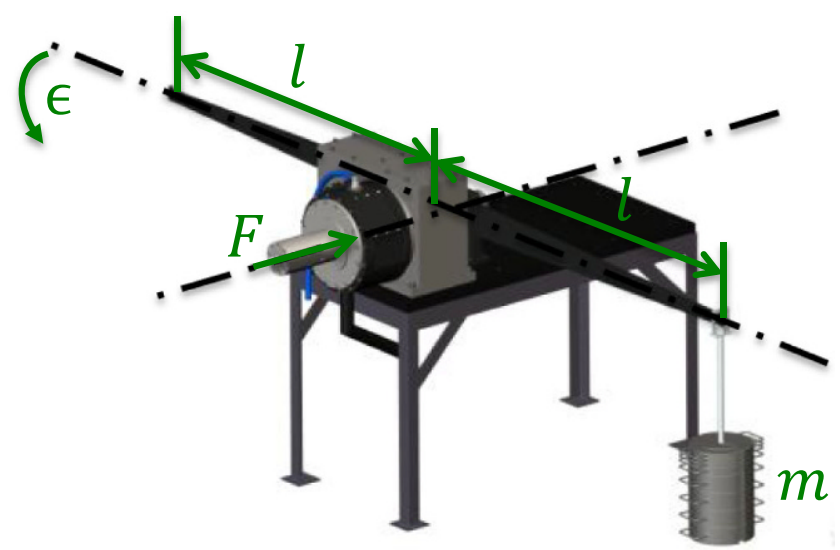

Abb. 4 Prüfstand LK3 (statisch) - schematisch [33]

\subsection{Betriebsarten}

Im Rahmen der Untersuchungen kommen drei Betriebsarten in jeweils unterschiedlichen Gleitgeschwindigkeitsbereichen zum Einsatz (Abb. 3).

\subsubsection{Kriechverhalten und Mikroschlupf bei konstanter Drehmomentbelastung}

Bei den Versuchen zur Haftreibungszahl sowie zum Reibungsverhalten bei Mikroschlupf wird der Prüfstand LK3 (statisch) verwendet (Abb. 4). Es werden hierbei Belastungsdrehmoment $T_{R}$ und Anpresskraft aufgebracht und die resultierende Winkelbewegung (Stillstand/Kriechrate) gemessen. Die Werte von Belastungsdrehmoment und Anpresskraft entsprechen einem Wert der ausgenutzten Reibungszahl $\mu_{\mathrm{a}}$ nach Gl. 1 .

$\mu_{a}(m, p, \varepsilon)=\frac{m \cdot g \cdot l \cdot \cos (\varepsilon)}{p \cdot A \cdot r_{m} \cdot z}$

$\mu_{a}[-] \quad$ Ausgenutzte Reibungszahl

$m[\mathrm{~kg}] \quad$ Masse d. Belastungsgewichts

$l[\mathrm{~m}] \quad$ Hebellänge

$\varepsilon\left[{ }^{\circ}\right] \quad$ Verdrehwinkel

$p\left[\mathrm{~N} / \mathrm{mm}^{2}\right] \quad$ Flächenpressung

$A\left[\mathrm{~mm}^{2}\right] \quad$ Reibfläche

$r_{m}[\mathrm{~mm}] \quad$ Mittl. Reibradius

$z$ [-] Reibflächenanzahl 


\subsubsection{Stationärer Langsamlaufschlupf}

Bei den Versuchsreihen mit definiertem Langsamlaufschlupf (Abb. 3 Mitte) wird die Kupplung im Stillstand geschlossen und mit definierter Axialkraft $\mathrm{F}_{\mathrm{ax}}$ beaufschlagt. Anschließend wird die Kupplung innerhalb einer definierten Drehzahlanstiegszeit $\Delta \mathrm{t}$ auf Zieldrehzahl $\mathrm{n}$ aufgerissen und eine definierte Zeit stationär bei dieser Schlupfdrehzahl betrieben.

\subsubsection{Lastschaltungen}

Bei Lastschaltungen wird die geöffnete Kupplung auf Differenzdrehzahl beschleunigt. Nach Erreichen der AusgangsDifferenzdrehzahl $\mathrm{n}_{\max }$ wird eine definierte Axialkraft $\mathrm{F}_{\mathrm{ax}}$ auf die Kupplung aufgebracht. Durch das sich aufbauende Reibmoment wird die Differenzdrehzahl abgebaut und bei Bremsversuchen die Kupplung bis auf Stillstand abgebremst (Abb. 3 rechts). Es ist zu beachten, dass eine Lastschaltung einen hochgradig instationären Reibvorgang darstellt.

\subsubsection{Konditionierung}

Vor den experimentellen Untersuchungen zum Reibungsverhalten werden die Versuchspakete über Lastschaltungen konditioniert und ein gleichmäßiger und stabiler Konditionierungszustand sichergestellt.

Es hat sich hierbei eine dreistufige Konditionierung als zielführend herausgestellt [33]. Die Konditionierung besteht aus einem Einlauf (angelehnt an [11]) und zusätzlichen Lastschaltungen bei erhöhten Öleinspritztemperaturen (Abb. 5).

\subsection{Versuchsablauf und -auswertung}

Die Versuchsreihen zum Reibungsverhalten werden mit konditionierten Versuchspaketen durchgeführt. Im Folgenden werden Versuchsablauf und -auswertung beschrieben.

\subsubsection{Stationärer Langsamlaufschlupf}

Ein Versuchsblock (Abb. 5) ist durch eine konstante Öleinspritztemperatur $\left(\vartheta_{\text {Öl }}\right)$ gekennzeichnet. Der spezifische Ölvolumenstrom $\dot{v}$ wird konstant gehalten. Vor und nach den Messreihen werden Lastschaltungen durchgeführt. Innerhalb der Versuchsreihen werden blockweise Flächenpressung (p), Gleitgeschwindigkeit $\left(\mathrm{v}_{\mathrm{g}}\right)$ und ggf. Drehrichtung (DR) variiert. Die Messpunkte werden mehrfach belegt. Für die Auswertung der Reibungszahl wird der stationäre Bereich der Messung herangezogen. Die ermittelten Reibungszahlen lassen sich über der Gleitgeschwindigkeit für z. B. verschiedene Öleinspritztemperaturen und Flächenpressungen darstellen [33].

\subsubsection{Haftreibung und Mikroschlupf}

Jeder Versuchsblock hier ist wieder durch eine feste Öltemperatur $\left(\vartheta_{\text {öl }}\right)$ gekennzeichnet. Es werden blockweise Flächenpressung (p) und ausgenutzte Reibungszahl $\left(\mu_{\mathrm{a}}\right)$ variiert. Die Messpunkte werden jeweils mehrfach belegt.

Aus dem Zeitverlauf des Verdrehwinkels wird eine mittlere Kriechrate $\dot{\varepsilon}=|(\Delta \varepsilon) /(\Delta \mathrm{t})|_{\mathrm{m}}$ in $\left(\begin{array}{l}\circ \\ \overline{\mathrm{h}}\end{array}\right)$ berechnet. Bei Variation der ausgenutzten Reibungszahl $\mu_{\mathrm{a}}$ ergeben sich unterschiedliche Kriechraten. Diese lassen sich in eine mittlere Gleitgeschwindigkeit umrechnen, wodurch die übliche Darstellung der Reibungszahl über der Gleitgeschwindigkeit und damit eine Einordnung zu den Ergebnissen der anderen Betriebsmodi möglich ist [33].
Abb. 5 Versuchsablauf - Versuchsreihen bei definiertem Langsamlaufschlupf [33]

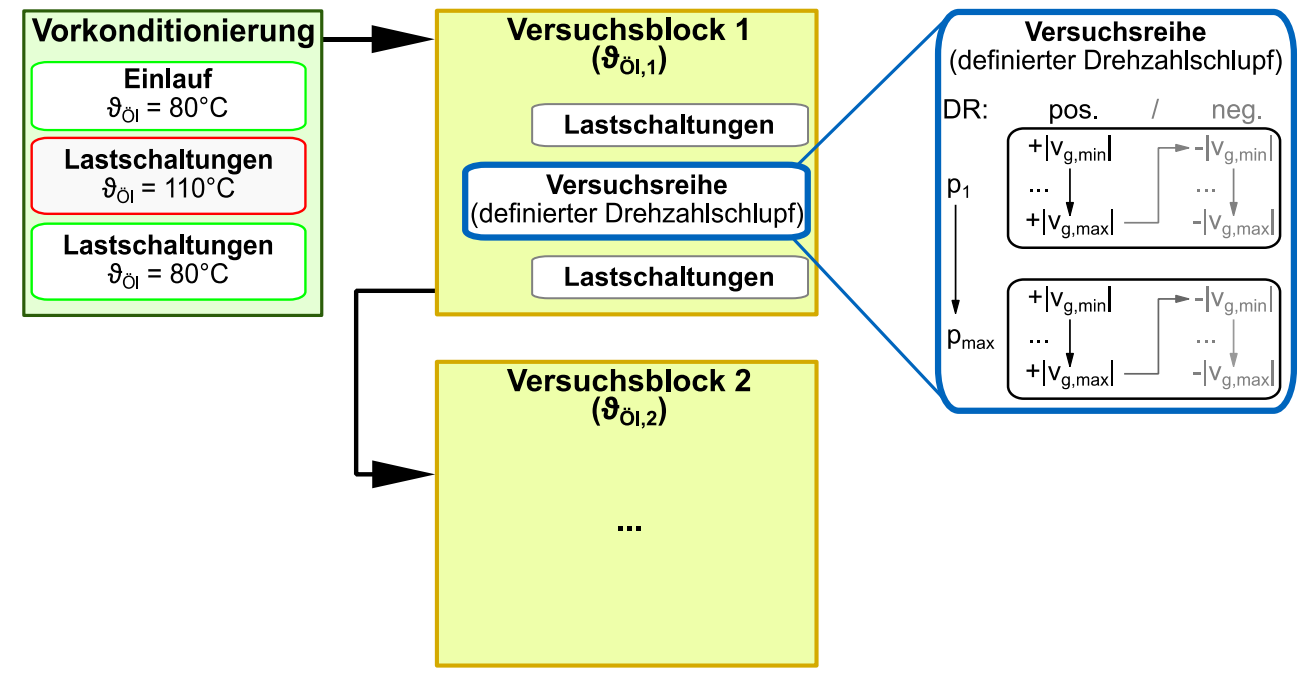


Abb. 6 Versuchsteile - Foto mit geometrischen Abmessungen [33]

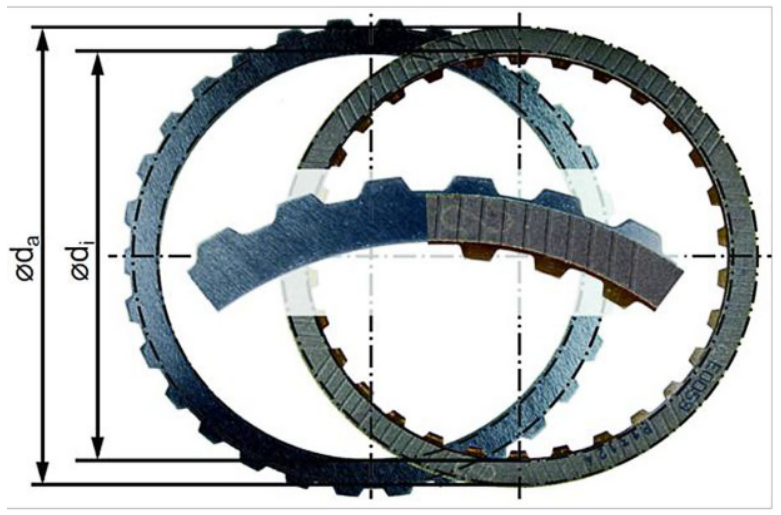

\begin{tabular}{|l|l|}
\hline $\begin{array}{l}\text { Reibflächeninnen- } \\
\text { durchmesser } \mathrm{d}_{\mathrm{i}}\end{array}$ & $165 \mathrm{~mm}$ \\
\hline $\begin{array}{l}\text { Reibflächenaußen- } \\
\text { durchmesser } \mathrm{d}_{\mathrm{a}}\end{array}$ & $188 \mathrm{~mm}$ \\
\hline mittlerer Reibradius $\mathrm{r}_{\mathrm{m}}$ & $88,2 \mathrm{~mm}$ \\
\hline Brutto-Reibfläche & $6229 \mathrm{~mm}^{2}$ \\
\hline
\end{tabular}

Abb. 7 Mikroschlupf und Haftreibung - ausgenutzte Reibungszahl über Gleitgeschwindigkeit. a $\mathrm{p}=0,5 \mathrm{~N} / \mathrm{mm}^{2}$, b $\mathrm{p}=1,0 \mathrm{~N} / \mathrm{mm}^{2}$ a

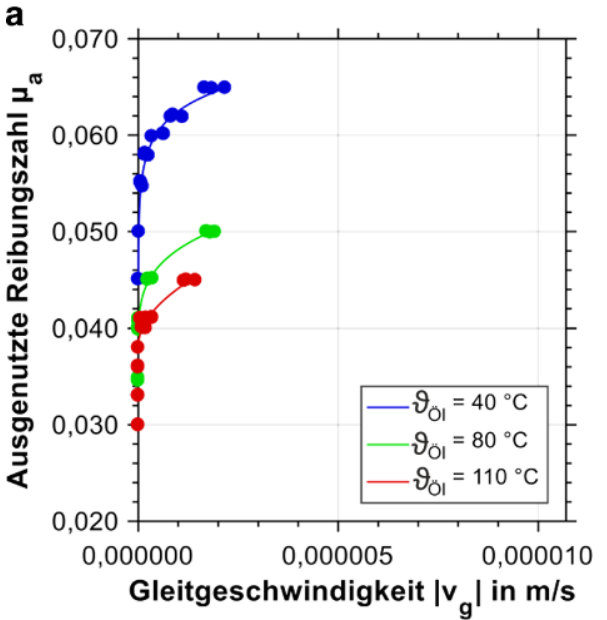

b

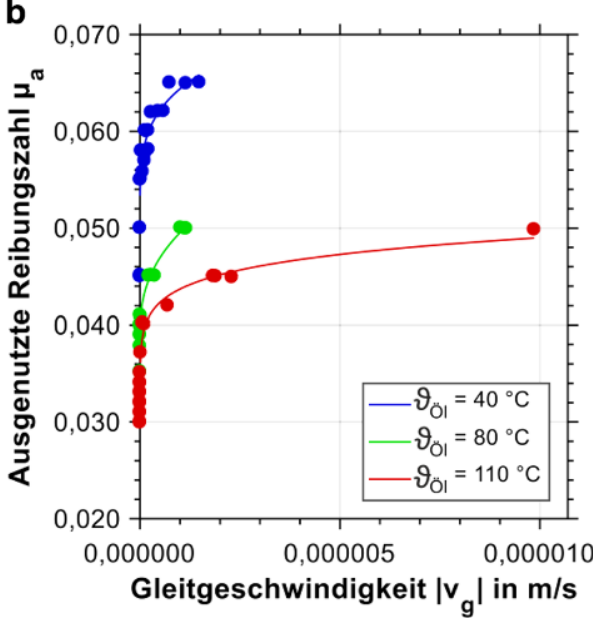

\section{Versuchsergebnisse zum Reibungsverhalten}

Nachfolgend werden exemplarisch Versuchsergebnisse zum Reibungsverhalten einer Lamellenkupplung aus einer $\mathrm{Au}-$ tomatikgetriebe-Anwendung aufgezeigt.

Die Kupplung ist aus wechselweise angeordneten Stahlund Belaglamellen aufgebaut. Die Stahllamellen weisen eine Kaltbandoberfläche (KBO) auf. Die Belaglamellen sind mit einem Papier-Reibbelag mit gruppenparallelem Nutbild versehen. Abb. 6 zeigt die Versuchslamellen und führt deren geometrischen Daten auf. Die experimentellen Untersuchungen werden an Kupplungspaketen mit sechs Reibflächen (vier Stahllamellen, drei Belaglamellen) durchgeführt.

Die Kupplungen werden mit einem typischen ATF-Serienschmierstoff betrieben. Tab. 1 führt die technischen Daten des Schmierstoffs auf.

Tab. 1 Schmierstoff - technische Daten [33]

\begin{tabular}{ll}
\hline Kinematische Viskosität bei $40^{\circ} \mathrm{C}$ & $26,8 \mathrm{~mm}^{2} / \mathrm{s}$ \\
Kinematische Viskosität bei $100^{\circ} \mathrm{C}$ & $5,6 \mathrm{~mm}^{2} / \mathrm{s}$ \\
Dichte bei $15^{\circ} \mathrm{C}$ & $840 \mathrm{~kg} / \mathrm{m}^{3}$ \\
\hline
\end{tabular}

\subsection{Haftreibung und Mikroschlupf}

Es wurden im Rahmen dieser Versuchsreihe Messungen bei drei Schmierstofftemperaturen mit Variation von ausgenutzter Reibungszahl sowie Flächenpressung durchgeführt. Es ist bei allen untersuchten Flächenpressungen und Schmierstofftemperaturen eine ausgenutzte Reibungszahl $\mathrm{zu}$ identifizieren, unterhalb der es zu keiner Relativverdrehung kommt. Bei Überschreiten der Haftreibungszahl kommt es zunächst zu Kriechen mit sehr niedrigen Kriechraten, die dann sehr schnell ansteigen.

In Abb. 7 ist die ausgenutzte Reibungszahl über der Gleitgeschwindigkeit für die untersuchten Schmierstofftemperaturen und Flächenpressungen dargestellt. Eine Ausgleichskurve vom Typ $\mathrm{f}\left(\mathrm{v}_{\mathrm{g}}\right)=\mathrm{a} \cdot \mathrm{x}^{\mathrm{b}}+\mathrm{c}$ mit $(0<\mathrm{b}<1)$ unterstützt die Visualisierung der Versuchsergebnisse. Es ist jeweils eine mit zunehmender Gleitgeschwindigkeit ansteigende Reibungszahl zu beobachten. Bei der höheren Flächenpressung von $1,0 \mathrm{~N} / \mathrm{mm}^{2}$ ist dieser Anstieg bei allen Schmierstofftemperaturen ausgeprägter als bei der niedrigeren Flächenpressung von $0,5 \mathrm{~N} / \mathrm{mm}^{2}$; grundsätzlich ist kein charakteristischer Unterschied der Reibungszahlen bei diesen Pressungen erkennbar. Der Übergang von 
Abb. 8 Stationärer Langsamlaufschlupf - Reibungszahl über Gleitgeschwindigkeit (Öleinspritztemperatur: $\vartheta_{\text {ÖI }}=40 / 80 / 110^{\circ} \mathrm{C}$, Spez. Ölvolumenstrom: $v=0,8 \mathrm{~mm}^{3} /\left(\mathrm{mm}^{2} / \mathrm{s}\right)$, Papierreibbelag - ATF, Flächenpressung: $\mathrm{p}=0,2 / 1,0 \mathrm{~N} / \mathrm{mm}^{2}$, Gleitgeschwindigkeit: $\mathrm{v}_{\mathrm{g}}=+(0,001 \ldots$ $0,200) \mathrm{m} / \mathrm{s}$ )

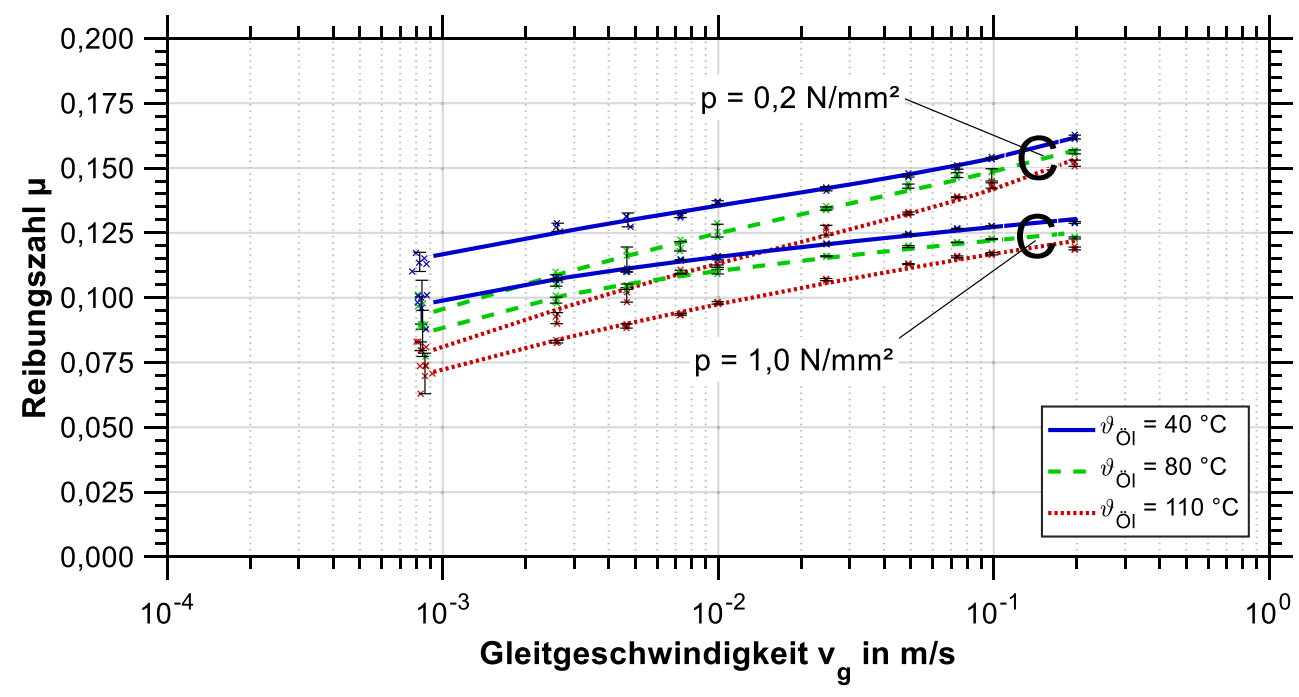

Bei Schmierstofftemperatur $40^{\circ} \mathrm{C}$ liegt der Haft-GleitÜbergang bei höherer Flächenpressung höher als bei niedriger Flächenpressung (Abb. 9a). Bei den höheren Schmierstofftemperaturen ist keine Abhängigkeit des HaftGleit-Überganges von der Flächenpressung erkennbar (Abb. 9b,c).

Für diese Reibmaterial-Schmierstoff-Paarung kann festgestellt werden, dass die ohne Kriechen bzw. bei sehr niedrigen Kriechraten übertragbaren Reibungszahlen deutlich unter den Reibungszahlen bei den Lastschaltungen und den Reibungszahlen bei stationärem Langsamlaufschlupf liegen.

\section{Zusammenfassung und Ausblick}

Kenntnisse zum übertragbaren Drehmoment nasslaufender Lamellenkupplungen bei den entsprechenden Belastungen und Betriebsbedingungen sind die Basis für eine betriebssichere Auslegung und Dimensionierung.

Im Rahmen grundlegender experimenteller Untersuchungen wurden das Reibungsverhalten bei Mikroschlupf sowie die übertragbare Haftreibungszahl untersucht. Es wurde eine Test- und Auswertemethodik entwickelt und auf ausgewählte Reib-/Schmierstoffpaarungen angewendet.

Die Ergebnisse für einen Papierreibbelag mit ATF zeigen, dass für diese Reibmaterial-Schmierstoff-Paarung die ohne Kriechen bzw. bei sehr niedrigen Kriechraten übertragbaren Reibungszahlen deutlich unter den Reibungszahlen aus den Lastschaltversuchen liegen. Wenn für die Dimensionierung von Schaltelementen mit Haltefunktion (z. B. Lamellenbremsen) Reibungszahlen aus dynamischen Untersuchungen oder Stationärschlupf-Versuchen herangezogen werden, ist mit einem deutlichen Kriechen zu rechnen. Die Erkenntnisse können einen Beitrag zur Erjeweils allmählich statt. 
Abb. 9 Reibungszahl über Gleitgeschwindigkeit - Gesamtdarstellung. a $\vartheta_{\mathrm{O}} \mathrm{O}=40^{\circ} \mathrm{C}$, b $\vartheta \ddot{O ̈}=80^{\circ} \mathrm{C}$, c $\vartheta_{\mathrm{ÖI}}=110^{\circ} \mathrm{C}$ (Schmierstoff-/Öleinspritztemperatur: $40 / 80 / 110^{\circ} \mathrm{C}$, Flächenpressung: $p=0,5(1,0) \mathrm{N} / \mathrm{mm}^{2}$, Papierreibbelag - ATF, Lastschaltung: Spez. Reibarbeit: $\mathrm{q}=0,35 \mathrm{~J} / \mathrm{mm}^{2}$, Max. spez. Reibleistung: $\mathrm{q}_{\mathrm{p} 0}=0,78 \mathrm{~W} / \mathrm{mm}^{2}$, Max. Gleitgeschwindigkeit: $\mathrm{v}_{\mathrm{g} 0}=13,0 \mathrm{~m} / \mathrm{s}$, Spez. Ölvolumenstrom: $\left.v=0,8 \mathrm{~mm}^{3} /\left(\mathrm{mm}^{2} / \mathrm{s}\right)\right)$

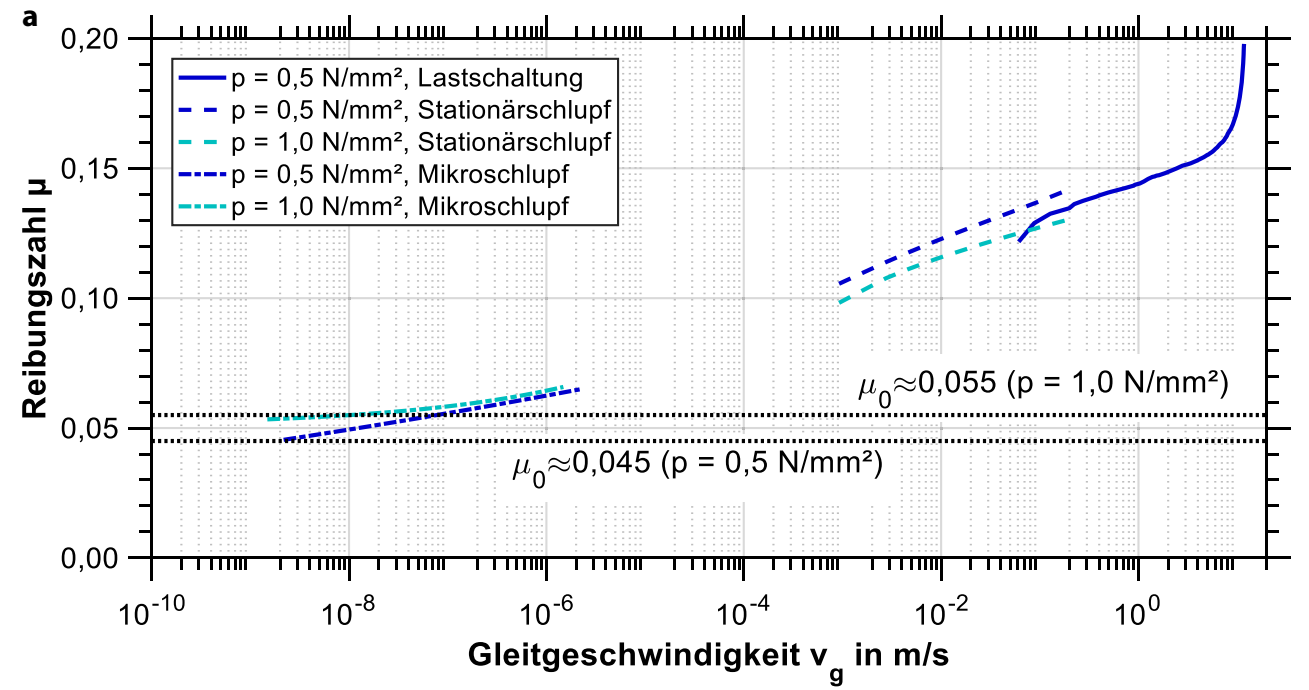

b
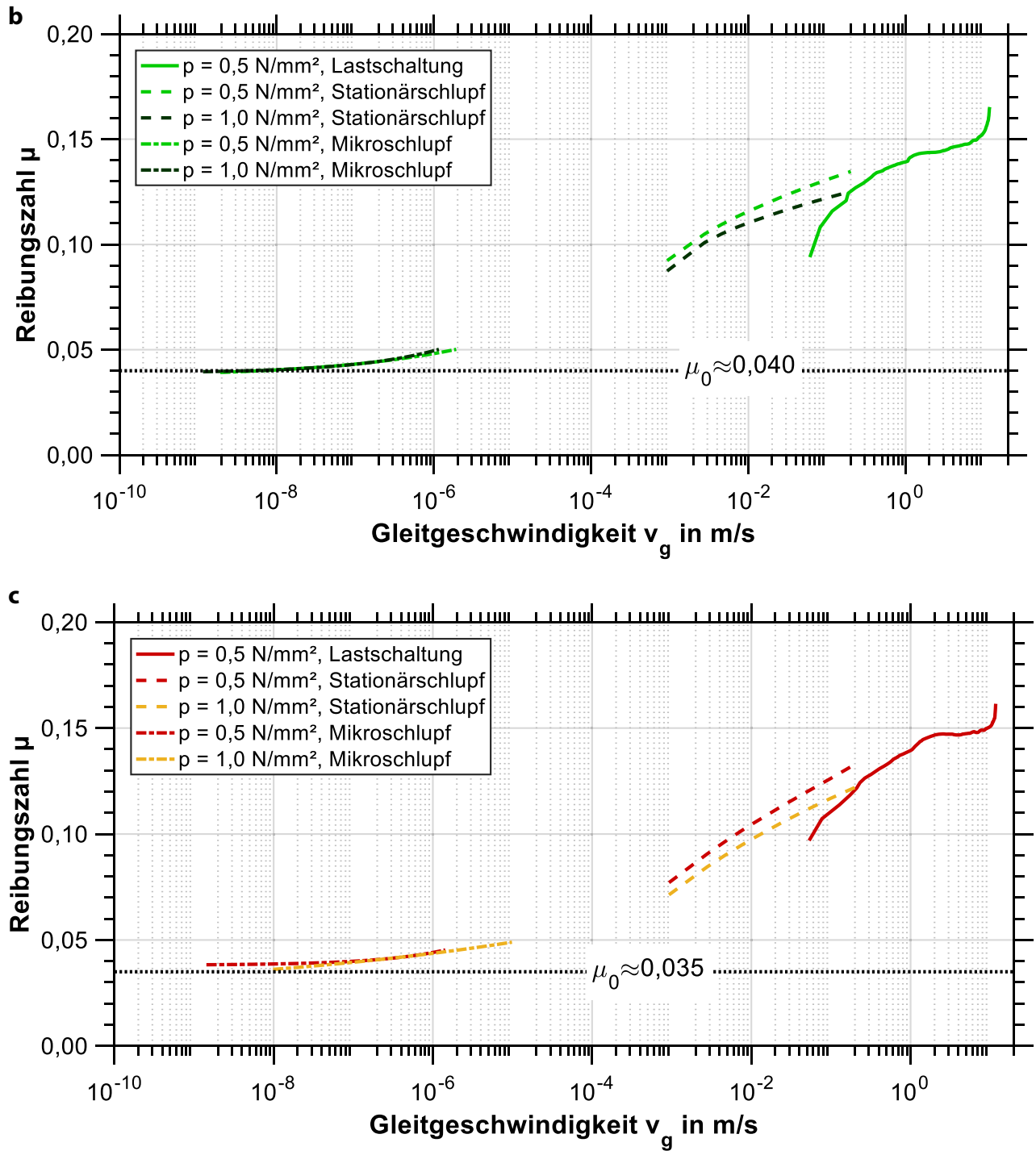
höhung der Dimensionierungs- und Auslegungssicherheit nasslaufender Lamellenkupplungen leisten.

Aktuell liegen jedoch noch keine systematischen Ergebnisse zu den Einflüssen von Schmierstoff, Baugröße und Reibbelag auf das Reibungsverhalten bei Langsamlaufund Mikroschlupf vor. Auch Einflüsse der Stahllamellenbeschaffenheit (z. B. Rauheit/Nitrierschicht) sowie Wellung der Belaglamellen bei diesen Betriebszuständen sind unbekannt. Anhand weitergehender Untersuchungen werden mit der gezeigten Versuchs- und Auswertemethodik systematisch Erkenntnisse zu diesen Einflüssen erarbeitet.

Funding The authors would like to thank for sponsorship and support received from the Research Association for Drive Technology e. V. (FVA) and the members of the project committee. The presented results are based on the research project FVA no. 719/I undertaken by the Research Association for Drive Technology e. V. (FVA). Das IGF-Vorhaben 18103N/1 der Forschungsvereinigung Antriebstechnik e. V. (FVA) wurde über die AiF im Rahmen des Programms zur Förderung der industriellen Gemeinschaftsforschung und -entwicklung (IGF) vom Bundesministerium für Wirtschaft und Energie aufgrund eines Beschlusses des Deutschen Bundestages gefördert. Die FVA übernimmt keine Gewähr für die Richtigkeit, die Genauigkeit und Vollständigkeit der Angaben sowie die Beachtung privater Rechte Dritter. Forschungsvereinigung Antriebstechnik e. V. (FVA), Lyoner Str. 18, 60528 Frankfurt/ Main.

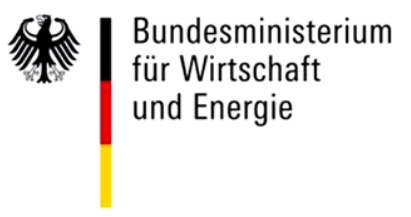

Funding Open Access funding enabled and organized by Projekt DEAL.

Interessenkonflikt K. Völkel, G.J. Meingaßner, H. Pflaum und K. Stahl geben an, dass kein Interessenkonflikt besteht.

Open Access Dieser Artikel wird unter der Creative Commons Namensnennung 4.0 International Lizenz veröffentlicht, welche die Nutzung, Vervielfältigung, Bearbeitung, Verbreitung und Wiedergabe in jeglichem Medium und Format erlaubt, sofern Sie den/die ursprünglichen Autor(en) und die Quelle ordnungsgemäß nennen, einen Link zur Creative Commons Lizenz beifügen und angeben, ob Änderungen vorgenommen wurden.

Die in diesem Artikel enthaltenen Bilder und sonstiges Drittmaterial unterliegen ebenfalls der genannten Creative Commons Lizenz, sofern sich aus der Abbildungslegende nichts anderes ergibt. Sofern das betreffende Material nicht unter der genannten Creative Commons Lizenz steht und die betreffende Handlung nicht nach gesetzlichen Vorschriften erlaubt ist, ist für die oben aufgeführten Weiterverwendungen des Materials die Einwilligung des jeweiligen Rechteinhabers einzuholen.

Weitere Details zur Lizenz entnehmen Sie bitte der Lizenzinformation auf http://creativecommons.org/licenses/by/4.0/deed.de.

\section{Literatur}

1. Heilenkötter D (1990) Untersuchungen zum Schlupfverhalten nasslaufender Lamellenkupplungen. Dissertation, Universität der Bundeswehr Hamburg

2. Lloyd F, Reffett T, Wyatt D (1994) Static friction-What it is, what affects it. SAE Tech Pap, Bd. 941034

3. Albers A, Stier C (2010) Analysis of geometrical deviations in clutch systems and their Interdependencies in relation to the excitation of judder vibrations. Proc of Fisita World Automot Congr, Budapest

4. Fidlin A, Stamm W (2009) On the radial dynamics of friction disks. Eur J Mech A Solids 28(3):526-534. https://doi.org/10. 1016/j.euromechsol.2008.12.001

5. Stamm W (2010) Stabilization of sliding motion by partial stiction. Proc Appl Math Mech 10(1):733-738. https://doi.org/10.1002/ pamm.201010350

6. Mäki R, Ganemi B, Höglund E, Olsson R (2007) Wet clutch transmission fluid for AWD differentials: influence of lubricant additives on friction characteristics. Lubr Sci 19:87-99. https://doi.org/ $10.1002 / 1 \mathrm{~s} .33$

7. Ingram M, Noles J, Watts R, Harris S, Spikes HA (2010) Frictional properties of automatic transmission fluids: part I-Measurement of friction-sliding speed behavior. Tribol Trans 54(1):145-153. https://doi.org/10.1080/10402004.2010.531888

8. Stockinger U, Mühlenstrodt K, Völkel K, Pflaum H, Lipinsky D, Stahl K, Arlinghaus HF (2019) Analyse tribologischer Schichten mit der Flugzeit-Sekundärionenmassenspektrometrie (ToF-SIMS) - Additiveinflüsse auf das Reibungsverhalten nasslaufender Lamellenkupplungen. Forsch Ingenieurwes 83:219-226. https://doi.org/ 10.1007/s10010-019-00305-0

9. Mosbach C (2002) Das Reibungs- und Reibschwingverhalten nasslaufender Lamellenkupplungen. Dissertation, Technische Universität München

10. Stockinger U, Pflaum H, Stahl K (2018) Friction screening tests for wet multi-plate clutches-Variation of lubricant and friction lining. Proc of Asia Int Conf on Trib, Kuching

11. Stockinger U, Pflaum H, Stahl K (2018) Zeiteffiziente Methodik zur Ermittlung des Reibungsverhaltens nasslaufender Lamellenkupplungen mit Carbon-Reibbelag. Forsch Ingenieurwes 82:1-7. https:// doi.org/10.1007/s10010-017-0253-x

12. Zou Q, Rao C, Barber G, Zhou B, Wang Y (2013) Investigation of surface characteristics and tribological behavior of clutch plate materials. Wear 302:1378-1383. https://doi.org/10.1016/j.wear.2012. 10.024

13. Ost W, De Baets P, Degrieck J (2001) The tribological behaviour of paper friction plates for wet clutch application investigated on SAE\# II and pin-on-disk test rigs. Wear 249:361-371

14. Geier N (2003) Untersuchung des Reibungs- und Verschleißverhaltens nasslaufender Kupplungen in Abhängigkeit ihrer Reibflächentopographie. Dissertation, Technische Universität München

15. Bäse MU, Dzimko M, Deters L (2016) Empirische Bewertung von Zusammenhängen zwischen Endbearbeitungsparametern und Reibungsverhalten von Lamellenreibpaarungen im Mikroschlupfbetrieb. 57. Tribol-Fachtag. Ges für Tribol e. V., Göttingen

16. Voelkel K, Pflaum H, Stahl K (2020) Running-in behavior of wet multi-plate clutches: introduction of a new test method for investigation and characterization. Chin J Mech Eng 33:34. https://doi. org/10.1186/s10033-020-00450-6

17. Berglund K, Marklund P, Larsson R, Olsson R (2015) Predicting boundary friction of aging limited slip differentials. J Tribol 137(1):12101. https://doi.org/10.1115/1.4028403

18. Ingram M, Noles J, Watts J, Harris S, Spikes HA (2010) Frictional properties of automatic transmission fluids: part II-Origins of 
friction-sliding speed behavior. Tribol Trans 54(1):154-167. https:// doi.org/10.1080/10402004.2010.531889

19. Voelkel K, Pflaum H, Stahl K (2019) Einflüsse der Stahllamelle auf das Einlaufverhalten von Lamellenkupplungen. Forsch Ingenieurwes 83:185-197. https://doi.org/10.1007/s10010-019-00303-2

20. Katsukawa M (2019) Effects of the physical properties of resins on friction performance. SAE Tech Pap, Bd. 2019-01-0341. https:// doi.org/10.4271/2019-01-0341

21. Höhn B-R, Pflaum H, Mosbach C (2003) Methodik zur Beurteilung des Schmierstoff-einflusses auf das Reibschwingverhalten nasslaufender Lamellenkupplungen. VDI-Fachtag „Kupplungen und Kupplungssysteme in Antrieben“. VDI Ber, Bd. 1786, S 455-468

22. Marklund P, Mäki R, Larsson R, Höglund E, Khonsari MM, Jang J (2007) Thermal influence on torque transfer of wet clutches in limited slip differential applications. Tribol Int 40:876-884. https:// doi.org/10.1016/j.triboint.2006.09.004

23. Mäki R, Ganemi B, Olsson R (2006) Wet clutch transmission fluid for AWD differentials: base fluid influence on friction characteristics. Tribotest 12:47-56. https://doi.org/10.1002/tt.4

24. Schneider T, Strebel M, Pflaum H, Stahl K (2019) Spontanschädigungsverhalten von nasslaufenden Lamellenkupplungen mit organischen und metallischen Reibbelägen. Forsch Ingenieurwes 83:199-207. https://doi.org/10.1007/s10010-019-00304-1

25. Meingaßner GJ, Pflaum H, Stahl K (2015) Test-rig based evaluation of performance data of wet disk clutches. Proc of 14th Int CTI Symp, Berlin
26. Frey D, Ast E, Brügel E (1998) European test procedures and test equipment for slip-controlled wet disc clutches. SAE Tech Pap, Bd. 982258. https://doi.org/10.4271/982258

27. Mäki R (2005) Wet clutch tribology: friction characteristics in limited slip differentials. Dissertation, Lulea University of Technology

28. Gao H, Barber GC, Chu H (2002) Friction characteristics of a paper-based friction material. Int J Automot Technol 3(4):171-176

29. Förster HJ (1991) Automatische Fahrzeuggetriebe - Grundlagen, Bauformen, Eigenschaften, Besonderheiten. Springer, Berlin, Heidelberg, New York

30. Gommel GS (1973) DKA-Reibwertprüfmaschine zur Prüfung von Ölen und Belagwerkstoffen, insbesondere für automatische Getriebe. Automob Techn Z 75:247

31. Herscovici S (1969) Determining the static and dynamic coefficient of friction and its causes for variation. SAE Tech Pap, Bd. 690570. https://doi.org/10.4271/690570

32. Meingaßner GJ, Pflaum H, Stahl K (2016) Drehmomentübertragungsverhalten nasslaufender Lamellenkupplungen bei geringen Differenzdrehzahlen. In: Proc of 4. Tribol.- u Schmierst.kongr. GETLUB

33. Meingaßner GJ (2017) Methodik zur Untersuchung des Reibungsverhaltens nasslaufender Lamellenkupplungen bei Langsamlaufund Mikroschlupf. Dissertation, Technische Universität München

34. Hämmerl B (1994) Lebensdauer- und Temperaturverhalten ölgekühlter Lamellenkupplungen bei Lastkollektivbeanspruchung. Dissertation, Technische Universität München 